\title{
EMERGENCIES IN GENERAL PRACTICE
}

\section{"THE BLEEDER"}

\author{
BY \\ R. G. MACFARLANE, M.A., M.D. \\ Clinical Pathologist and Radcliffe Lecturer in Haematology, Radcliffe Infirmary, Oxford
}

The first experience of bleeding which cannot be controlled by conventional treatment is alarming for both patient and doctor. This alarm, which is seldom justified by any immediate danger of exsanguination, is largely due to what seems an unnatural occurrence. We are so used to the fact that minor injuries stop bleeding spontaneously that automatic haemostasis is taken for granted. Yet, since the circulating blood is subject to the ordinary laws of hydrostatics, it is actually one of the many biological mysteries.

The mechanism responsible is only partly understood and is of great complexity. Its components are the coagulation of the blood, the contraction of injured vessels, and the adhesion of the platelets, these events being probably closely integrated and to some extent interdependent. The important fact is that any serious defect in their operation will result in prolonged bleeding from even trivial injuries, and only when attempts are made to arrest this is it realized how dependent we are upon the normal mechanism. Dressings, tight bandages, plugging, and sutures are all useless; the blood seeps through them as if it were water, or, worse, if its free escape is prevented it infiltrates the tissues. Bleeding can be arrested by the application of pressure to cut off the blood supply to the injured area, but this cannot be maintained for long without causing necrosis and an extension of the area of bleeding. The usual methods for arresting haemorrhage act only by assisting the physiological mechanism; they cannot replace it.

Temporary pressure is normally useful, as it allows time for vessels to contract and for a firm clot to form. Dressings accelerate normal clotting by providing a large surface and also reinforce the clot; sutures close a wound cavity, so that the clot has the greatest occlusive effect. But if the clot fails to form, or the vessels to contract, these measures are ineffective and may be positively dangerous. It is therefore important for the doctor to recognize when he is dealing with haemorrhage due to these failures, and it must be emphasized that, unless the bleeding can be readily controlled by the methods described in this article, the patient should be transferred to hospital without delay. The recent establishment of regional "haemophilia centres" for the diagnosis and treatment of the haemorrhagic states is likely to assist greatly in the efficient hospital treatment of these conditions.*

\section{Diagnosis}

Fortunately, bleeders, or those of them who bleed dangerously on slight provocation, are rare. Haemophilic subjects, who, because of the severity of their disability, form the most important group, occur in the proportion of only 1 or 2 per 100,000 of the population of this country. But there are other conditions which cause abnormal bleeding, and, since their appropriate treatment differs, the doctor should know what sort of case he has to deal with.

*See British Medical Journal, 1954, 2, 1287.
Briefly, the haemorrhagic states can be divided into those due to defective coagulation and those due to abnormalities involving the vessels or platelets; these two groups can each be subdivided into constitutional conditions, often hereditary, and acquired conditions, often secondary to some underlying disease.

It must be first decided whether excessive bleeding is merely due to a locally overtaxed but otherwise normal haemostatic system or to one of these haemorrhagic states. Persistent bleeding may occasionally occur in normal people; after tooth extraction, for example, it may go on for perhaps 24 hours; epistaxis due to local vascular erosion may be prolonged or frequently repeated; secondary haemorrhage from infected wounds is due to proteolytic digestion of blood clot and tissues; and in uterine bleeding the bounds of normal are notoriously hard to define. But these episodes can usually be distinguished from the bleeding of the haemorrhagic states by the patient's history, and from certain features of the bleeding itself. If the patient has experienced previous injuries or operations such as tonsillectomy without serious haemorrhage, he is unlikely to have a constitutional haemorrhagic state.

The genuine bleeder almost always has a long history, often going back to infancy, of prolonged bleeding not limited to one particular part of the body, but from any minor injury or operation site, and in the form of epistaxis, gastro-intestinal bleeding, easy bruising, purpura, or the formation of haematomas and swellings of the deep tissues and joints, and so on. A point of diagnostic value is the occurrence of similar troubles in other members of the family. Symptoms which might be equivocal by themselves become significant if they also occur in blood relations.

The bleeding itself may give information on the type of defect which has caused it. In cases of defective clotting the vessels are usually normal, and bleeding does not occur unless they have been damaged. In such cases, following an injury which might have been thought trivial by ordinary standards, there is often only slight bleeding at first which may even cease for some hours; it then begins again and becomes uncontrollable, sometimes continuing for days and even weeks. Haemorrhage into the tissues occurs usually after a blow or strain, producing massive haematomata involving any part of the body, particularly the muscles and joint cavities.

Bleeding due to vascular or platelet abnormalities, on the other hand, is often spontaneous and is mainly limited to the skin and mucous membranes. Petechiae and ecchymoses appear without obvious cause; bleeding from the nose and gums is common, being a diffuse oozing without any definite bleeding point; gastro-intestinal bleeding and menorrhagia may be dangerously profuse. It is characteristic of these purpuric conditions that even small needle punctures bleed persistently.

Further differentiation may be obtained by considering the characteristics of some of the different members of the two groups of haemorrhagic conditions, but only the commoner conditions are described here.

All types of clotting disorders tend to produce a similar symptomatology. In the constitutional group both haemophilia and Christmas disease (also called haemophilia $\mathbf{A}$ and haemophilia $B$ respectively) are inherited as sex-linked 
recessive conditions, affecting only males and being transmitted through apparently normal females. They cannot be distinguished by clinical findings alone.

In the group of acquired clotting defects there is a haemophilia-like state occurring in females which is due to an anticoagulant in the blood, developed during or after pregnancy, perhaps as an immune reaction. An acute fibrinogen deficiency may also occur with disastrous rapidity in some cases of premature detachment of the placenta, with uncontrollable uterine and sometimes generalized bleeding. It is due to the intravascular entry of placental fragments which cause the removal of fibrinogen from the circulating blood. Lastly, the widespread use of the coumarin drugs in the treatment of thrombosis produces a few cases of overdosage, with external and internal haemorrhage due to factor 7 and prothrombin deficiency.

Of the purpuric conditions the commonest is secondary thrombocytopenic purpura, which is often due to drug sensitivity, leukaemia, aplastic anaemia, or carcinomatosis. In a smaller group of cases the thrombocytopenia is apparently essential.

\section{Principles of Local Treatment of External Bleeding}

When the bleeding point is accessible local attempts can be made to produce a firm clot. However, even the most rapid clotting cannot extend against a flow of blood, since the fibrin will be washed away from the bleeding point. It is therefore essential to arrest the blood flow by pressure or by stimulating vascular contraction. If clotting is defective it 'will also be necessary to apply a coagulant, of which the best is thrombin. If used in sufficient concentration, solutions of thrombin will clot in a few seconds any blood that contains fibrinogen. Another useful coagulant is Russell's viper venom. It is more active than thrombin in low concentrations, except in cases of prothrombin deficiency, but like thrombin will not clot blood lacking fibrinogen.

The best way of applying these coagulants is by means of a dressing saturated with the solution. But as ordinary gauze or cotton-wool packs have eventually to be removed and this often restarts the bleeding, an absorbable dressing such as "oxycel" or "calgitex" should be used. These become pliable and adhesive when moistened, and can be accurately moulded to the bleeding point and left in position until digested.

\section{Treatment of Specific Haemorrhages Epistaxis}

Since epistaxis often stops spontaneously drastic measures should not be undertaken unless the blood loss is serious. In cases of defective clotting the bleeding point is likely to be on the anterior part of the septum, where it may be seen and dealt with directly. A pad of oxycel or calgitex gauze, a few millimetres square, should be soaked with thrombin or viper venom, applied to the bleeding area and held there by the gentle pressure of the flat handle of a scalpel or some similar instrument. After four to five minutes the instrument is removed, leaving the dressing adhering to the bleeding site. If this is effective the dressing should be left alone until it comes away naturally. If it fails, the process should be repeated, but great care should be taken not to damage the membranes of the nose and start new areas of bleeding. Thermal or chemical cauterization should never be used in these cases. If the bleeding point cannot be seen, a mixture of thrombin solution and $1 / 1,000$ adrenaline in equal parts can be sprayed into the nose with an ordinary atomizer; the anterior nares are then plugged with gauze and the patient holds his head forward, so that a firm clot forms in the nasal cavity. He should refrain from swallowing during this proceeding, or the clot may be dislodged. No attempt should be made to plug the posterior nares. If bleeding is not stopped further local treatment should be abandoned, since it is likely to do more harm than good. The patient should lie so that the blood does not run back into his throat and cause vomiting, and often bleed- ing will cease before a dangerous amount of blood has been lost. If it still continues, arrangements should be made for transfer to hospital.

Epistaxis in the purpuric conditions is usually a diffuse oozing which is difficult to control but seldom profuse enough to be dangerous. Plugging is often useless, and fresh bleeding points may be started at the slightest touch. A thrombin-adrenaline spray may be effective, and an ice pack applied to the nose may also be tried.

\section{Bleeding Tooth Sockets}

Dental extraction occasionally reveals for the first time that the patient is a bleeder. It need hardly be emphasized that good conservative dentistry is particularly important for such patients; unfortunately their teeth are often grossly and needlessly neglected.

In dealing with tooth sockets the object is not merely to stop the bleeding but to avoid delaying healing, since haemorrhage is likely to recur until this is complete. Tight plugging should not be used, therefore, as it causes eversion of the socket, nor clamping of the jaw with tight bandages, which may cause necrosis of the gum, nor caustics and styptics, as they destroy the tissues. In cases of defective clotting the gum margins should never be sewn together, since the blood, unable to escape, may infiltrate the tissues of the tongue and throat and cause fatal asphyxia.

A method which is applicable to almost all haemorrhagic states is as follows. A pledget of oxycel or calgitex, about the size of a marble, is soaked with thrombin or venom solution, and moulded into the shape of a mushroom. Any loose blood clots are washed away from the socket and the stalk of the "mushroom" is lightly pressed down into the cavity, the head fitting over the edges of the socket. A cotton-gauze swab or pack of the size required to fill the gap between the socket and the opposing teeth is then applied over the dressing and firmly pressed into position by the thumb and forefinger, which should also squeeze the edges of the socket together. This pressure must be maintained for at least five minutes and relaxed without dislodging the cotton pack. The patient can then close his jaws and if the pack is properly placed the bite should hold it in position without excessive pressure. In almost every case this procedure will stop the bleeding for several hours at least, though it is then apt to restart owing to digestion of the clot. It is a great advantage to have a small dental splint made which, better than the cotton pack, will hold the coagulant dressing firmly in place. Dressing with new applications of coagulant can be repeated once or twice in the 24 hours as required, but no attempts more drastic than these should be undertaken to control the bleeding. If it becomes dangerous the patient will require admission to hospital and transfusion.

\section{Open Wounds}

In cases of defective clotting, an open wound should be treated in much the same way as a tooth socket. It should be cleaned carefully and, if the edges fall together naturally, irrigated with thrombin or venom solution, and a pad of oxycel or calgitex, soaked with coagulant, laid over it. This is then covered with cotton gauze bandaged firmly in place, and manual pressure applied for five minutes. If bleeding recurs a repetition of the process should be carried out. If the edges of the wound tend to gape they can be partly drawn together by strapping, after lightly packing and covering with absorbable dressing and coagulant. The strapping should not be so tight as to prevent the escape of blood to the surface. When healing is complete the strapping can be removed without damaging the skin, by soaking it in a mixture of equal parts of liquid paraffin and petroleum ether. Wounds should not be stitched in cases of haemophilia or Christmas disease, though it may be necessary to ligate large bleeding vessels. Prolonged pressure, cauterization, or diathermy should never be employed, as subsequent sloughing inevitably causes bleeding from a larger area than before. If injections of tetanus antitoxin or antibiotics are 
considered essential they should not be given intramuscularly since they may cause serious local haemorrhage. Intravenous injections can be made with less risk of bleeding, but the danger of anaphylaxis must always be remembered.

In the purpuric conditions bleeding from skin wounds can usually be controlled by temporary pressure and a firm dressing. There is not the same danger of haematoma formation in these states, so that suturing is not contraindicated.

\section{Internal Bleeding : Deep-Tissue Haemorrhages}

Extensive bleeding into the tissues is common in haemophilia and its related conditions, and may involve a whole limb or half the trunk. Serious pressure effects and dangerous loss of blood may therefore occur. Unfortunately little can be done to limit these haemorrhages. As soon as it becomes obvious that bleeding is taking place the part should be put at rest and elevated if possible ; local application of ice packs has been recommended. Pressure dressings are not of much use unless the haematoma is small and is overlying bone; a haematoma of the scalp can be restricted, for instance, by a pad of "sorbo" rubber and a firm bandage. Haematomata should never be incised; the blood is almost always interstitial and cannot be evacuated by surgical means, and the incision itself becomes a new source of bleeding.

The most dangerous site for tissue bleeding is into the tongue and throat, because any swelling here interferes with the airway, sometimes with devastating rapidity. As soon as it is diagnosed arrangements should be made for the immediate transfer of the patient to hospital, where facilities are available to pass an endotracheal tube. The doctor must travel with him and be ready to pass an oral airway (as used by anaesthetists) to separate the tongue from the posterior pharyngeal wall, or failing this to pass a shortened rubber endotracheal tube through the nose, so that the tip passes beyond the obstruction and lodges at the level of the entrance to the larynx. If respiratory obstruction threatens it is a misplaced kindness to give morphine to relieve the patient's distress. His survival for the time being depends on vigorous respiratory efforts on his part, and the administration of any respiratory depressant can have fatal results.

Bleeding into the spinal cord, with signs of increasing compression, may occur, and demands immediate admission of the patient to hospital. Diagnostic difficulties are produced by retroperitoneal haemorrhages which may cause abdominal pain, tenderness, rigidity, vomiting, and fever. Such cases have been frequently misdiagnosed as appendicitis or a perforated ulcer, and the significance of abdominal symptoms in a patient known to be suffering from a haemorrhagic state has therefore to be considered with great care. Operation is justified only if it is certain the patient will die without it.

Haematemesis should be treated in the normal way. Haematuria, which may sometimes be prolonged for many days in patients with haemophilia and Christmas disease, can be treated only by rest and transfusions.

Haemarthroses are frequent in haemophilia and its related conditions, and the joint destruction which follows is the cause of much disability. In the acute phase the joint is extremely painful and morphine may have to be given, although non-habit-forming analgesics are preferable. The affected joint should be elevated and immobilized in a slightly flexed position. Ice packs are sometimes comforting.

\section{General Treatment}

\section{Haemophilia and Related Conditions}

Correction of the specific clotting defects cannot easily be carried out in the patient's own home. In haemophilia the blood defect can be temporarily corrected by transfusions of several pints of normal blood or plasma, which must be given within a few hours of its collection from the donor, as the active factor is very labile. Concentrated preparations derived from human and, quite recently, from bovine blood are being tested clinically, and it may be that in the near future they may be made generally available. If this hope is fulfilled then many of the local measures described here for the treatment of haemophilia will happily become obsolete.

In other clotting defects it is probable that amounts of fresh blood similar to those now necessary in haemophilia are required for efficient treatment; a possible exception is Christmas disease, in which it appears that stored blood or serum may be effective and that relatively smaller amounts are needed.

Apart from its effect on clotting, the patient may need a transfusion to restore a dangerously depleted blood volume, possibly before removal to hospital. If the blood pressure is falling alarmingly, the practitioner himself may be able to give a transfusion of 1 or 2 pints (about a litre) of reconstituted dried human plasma, or 1 or 2 pints of dextran solution, neither of which involves grouping or cross-matching, but a blood sample for grouping and cross-matching must be taken before giving dextran, as this substance interferes with grouping technique. Cutting down on a vein must be avoided if humanly possible ; destruction of available veins by irresponsible transfusion technique may cost the patient his life when another transfusion is needed. No general treatment of the constitutional clotting defects other than blood transfusion or the injection of the specific blood fractions has been found to be effective.

\section{Acquired Clotting Defects}

In cases of haemorrhage due to overdosage of one of the dicoumarin group of drugs, immediate contact should be made with the hospital which is undertaking laboratory control of the patient's treatment. An adequate dose of vitamin $\mathrm{K}_{1}$ should be given as soon as possible. An oral preparation is "konakion," which acts in a few hours. If the doctor is in the habit of collecting blood samples for prothrombin determination he should take a sample before giving the vitamin $K_{1}$.

Bleeding due to fibrinogen deficiency or the acute defibrination syndrome which may rarely complicate cases of premature detachment of the placenta must be treated with massive blood transfusion and, if it can be obtained, by injecting intravenously about $2 \mathrm{~g}$. of human fibrinogen in solution.

\section{The Purpura Group}

In thrombocytopenic purpura the first essential to general treatment is diagnosis, which in most cases requires laboratory investigation. Leukaemia, aplastic anaemia, sensitivity to drugs such as "sedormid," quinine, or even aspirin, or to certain chemicals, are the commonest causes, and these, when identified, must be treated by the appropriate means. In idiopathic thrombocytopenic purpura splenectomy gives the best chances of recovery. If splenectomy fails, or as a preparation for operation, a course of cortisone sometimes leads to considerable clinical improvement. Purpuric bleeding may occasionally be arrested by transfusion of fresh blood.

I have discussed the relevant parts of the treatment described in this article with Sir Robert Macintosh and Mr. G. Livingstone, of the Departments of Anaesthetics and Otolaryngology, Radcliffe Infirmary, and Dr. J. Grant, of the Oxford Regional Blood Transfusion Centre. I am most grateful for the advice they have given me.

Next article on Emergencies in General Practice."Antepartum Haemorrhage," by Mr. F. Stabler.

Refresher Course Book.-Copies of the second volume of collected articles from the Refresher Course for General Practitioners published in the Journal are still available at 25s. (postage 1s.) each. The volume contains 60 articles, all of which were revised by their authors for publication in the book. The first volume is now sold out and out of print. Copies of the second volume may be obtained from the Publishing Manager, B.M.A. House, Tavistock Square, London, W.C.1, or through any bookseller. 